\title{
Palliative care clinical rotations among undergraduate and postgraduate medical trainees in Canada: a descriptive study
}

\author{
Bruno Gagnon MD MSc, Anne Boyle MD, Fabienne Jolicoeur MSc, Mauranne Labonté MSc, \\ Kim Taylor MSc, James Downar MDCM MHSc
}

\section{Abstract}

Background: The number of medical undergraduate and postgraduate students completing palliative care clinical rotations in Canadian medical schools is currently unknown. The aim of this study was to assess the proportion of Canadian medical trainees completing clinical rotations in palliative care and to determine whether changes took place between 2008 and 2018.

Methods: In this descriptive study, all Canadian medical schools $(n=17)$ were invited to provide data at the undergraduate and postgraduate levels (2007/08-2015/16 and 2007/08-2017/18, respectively). Information collected included the number, type and length of palliative care clinical rotations offered and the total number of medical trainees or residents enrolled at each school.

Results: All 17 Canadian medical schools responded to the request for information. At the undergraduate level, palliative care clinical rotations were not offered in 2 schools, mandatory in 2 and optional in 13 . Three schools that offered optional rotations were unable to provide complete data and were therefore excluded from further analyses. In $2015 / 16$, only $29.7 \%$ of undergraduate medical students completed palliative care clinical rotations, yet this was a significant improvement compared to $2011 / 12(13.6 \%, p=$ 0.02). At the postgraduate level, on average, $57.9 \%$ of family medicine trainees completed such rotations between $2007 / 08$ and $2016 / 17$. During the same period, palliative care clinical rotations were completed by trainees in specialty or subspecialty programs in anesthesiology (34.2\%), geriatric medicine (64.4\%), internal medicine (30.9\%), neurology (28.2\%) and psychiatry (64.5\%).

Interpretation: Between 2008 and 2018, a large proportion of Canadian medical trainees graduated without the benefit of a clinical rotation in palliative care. Without dedicated clinical exposure to palliative care, many physicians will enter practice without vital palliative care competencies.

$\mathrm{P}$ alliative care can be appropriate at any age and any stage of an incurable disease or of an advanced lifethreatening illness, and can be provided along with curative treatment. ${ }^{1}$ It aims to give patients and those close to them the support they need to achieve the best possible quality of life..$^{2-5}$ Delivery of palliative care is a shared responsibility among all health care professions within all fields of medicine. However, only $25 \%-30 \%$ of practising physicians feel comfortable providing palliative care, ${ }^{6}$ and there is a shortage of trained palliative care providers in the workforce. ${ }^{7}$ In August 2013, the Canadian Medical Association adopted a resolution requesting that "all Canadian faculties of medicine create a curriculum for training in palliative care suitable for physicians at all stages of their medical education and relevant to the settings in which they practise."

To meet current and projected palliative care needs of Canadians, most physicians should receive clinical training in palliative care, ${ }^{8}$ and clinical rotations are an essential component of this. The proportion of medical undergraduate and postgraduate students completing palliative care clinical rotations in Canadian medical schools is unknown. To evaluate the status of palliative care clinical training in Canada, we aimed to estimate the proportion of Canadian medical trainees completing clinical rotations in palliative care and to determine whether changes in palliative care clinical training have recently taken place.

\section{Competing interests: None declared.}

This article has been peer reviewed.

Correspondence to: Bruno Gagnon, Gagnon.bruno@crchudequebec. ulaval.ca

CMAJ Open 2020. DOI:10.9778/cmajo.20190138 


\section{Methods}

\section{Setting and design}

This was a descriptive study conducted between July 2016 and June 2018 in which all Canadian medical schools were invited to participate. There are 17 medical schools in Canada, distributed across 5 geographic regions: British Columbia $(n=1)$, Prairies $(n=4)$, Ontario $(n=6)$, Quebec $(n=4)$ and Atlantic Canada $(n=2)$. Medical training consists of undergraduate training (3-5 yr) leading to an M.D. degree, followed by postgraduate training ( $\geq 2 \mathrm{yr}$ ).

\section{Data sources}

We asked members of the Canadian Society of Palliative Care Physicians' undergraduate and postgraduate training committees to identify a representative responsible for palliative care education from each medical school to complete the data collection form (Appendix 1, available at www.cmajopen.ca/ content/8/2/E257/suppl/DC1). One of the authors (F.J.) had multiple telephone communications with each school representative to provide support in completing the form.

Medical schools were asked to provide data for academic years 2007/08-2015/16 for undergraduate students, and 2007/08-2017/18 for postgraduate students. The following information was collected: type of palliative care clinical rotations offered (mandatory, optional), length of palliative care clinical rotations offered (in weeks), number of medical students enrolled each year, number of medical students who completed palliative care clinical rotations each year, whether the school offered an Enhanced Skills program in palliative care, number of residents who completed Enhanced Skills programs in palliative care each year, and number of residents who completed palliative care clinical rotations each year, by program specialty or subspecialty.

To determine the number of Canadian medical schools that offered mandatory or optional palliative care clinical rotations at the undergraduate level, we used data from 3 sources: information about the number of clinical rotations performed by students provided by the responding medical schools; for the nonresponding schools, curriculum information found on their faculty website, if available; and, if this information was not available, reports from the Association of Faculties of Medicine of Canada. ${ }^{9}$

We used reports from the Canadian Post-M.D. Education Registry ${ }^{10}$ and the Association of Faculties of Medicine of Canada $^{9}$ as a standardized data source to determine the number of medical students and residents enrolled each year between 2007/08 and 2017/18. We also obtained data for a selected group of specialty and subspecialty training programs for which we considered palliative care to be particularly relevant (i.e., anesthesiology, family medicine, geriatric medicine, internal medicine, neurology and psychiatry).

\section{Statistical analysis}

The data collection form was intended to collect data at the individual trainee level. Some of the responding schools used it, but others provided data in a different format, so the data had to be extracted by the research team. Owing to these format differences, only yearly and by-program aggregated analysis was possible. This allowed an estimate of the proportion of medical trainees who completed a clinical rotation from a given program in a given year. If trainees completed more than 1 rotation in palliative care, each individual rotation was counted. For each subgroup, the proportion of medical trainees for whom data were available over the total of medical trainees in Canada for this specific year was expressed as a percentage.

We defined complete data as the exact number of palliative care clinical rotations by year at both levels and by specialty at the postgraduate level. Nonresponding medical schools or medical schools unable to provide data on the number of palliative care clinical rotations were treated as missing data and were excluded from the analysis.

Data from medical schools were anonymized before being analyzed with Microsoft Excel 2013. We analyzed the increase in palliative care clinical rotations over time with the exact Wilcoxon 2-sample test using SAS 9.4 (SAS Institute).

\section{Postgraduate clinical rotations}

We calculated the estimated proportion of postgraduate trainees who completed a dedicated palliative care clinical rotation in a specific program or medical school by dividing the number of palliative care clinical rotations completed during a specific training year in the program or medical school by the total number of trainees enrolled in the program or medical school that year, then multiplying by the duration (in years) of the training program. For example, during the academic year $2009 / 10$, for the 5 -year anesthesiology program, 22 palliative care clinical rotations were completed among a total of 305 residents from the 9 responding schools that offered this residency program, leading to an estimated percentage of palliative care clinical rotations of $36.1 \%\left([22 / 305]^{*} 5\right)$. The estimated percentage could exceed $100 \%$ if, on average, trainees in a given program completed more than 1 palliative care rotation in a given year.

\section{Ethics approval}

This study did not require formal ethical approval, as determined by the Research Ethics Review Board of the Centre hospitalier universitaire de Québec-Université Laval. Of the 17 medical schools, 16 agreed with this determination. The remaining site (University of Toronto) received institutional ethical approval.

\section{Results}

All 17 Canadian medical schools responded to the request for information and were able to confirm whether they offered palliative care clinical rotations within their institution. Nine schools used the data collection form.

\section{Undergraduate trainees}

Of the 17 schools, 2 (12\%) did not offer any palliative care clinical rotations to their undergraduate students between 
2007/08 and 2015/16, 13 (76\%) offered rotations on an optional basis, and 2 (12\%) included palliative care clinical rotations on a mandatory basis (starting in 2015 in 1 case). However, 3 of the 13 schools that offered optional rotations were unable to provide complete data and were therefore excluded from further analyses. The clinical rotations varied from 1 to 4 weeks among and within schools; some schools were unable to provide this information. Therefore, we did not compare the duration of the rotations between schools.

Among the 14 Canadian medical schools that provided complete data about their undergraduate medical students, an estimated 665 (29.7\%) of 2239 students completed a clinical rotation in palliative care in 2015/16. Of the 665 students, 447 $(67.2 \%)$ were from the 2 medical schools with mandatory palliative care rotations, 1 of which started to offer mandatory palliative care clinical rotations in $2011 / 12$, and the other in 2014/15.

The estimated proportion of undergraduate trainees who completed palliative care clinical rotations increased significantly between $2011 / 12$ and $2015 / 16$, from $13.6 \%$ to $29.7 \%(p=0.02)$ for all responding schools, and from $5.9 \%$ to $12.7 \%(p=0.03)$ when the 2 schools with mandatory palliative care clinical rotations were excluded (Figure 1). In $2015 / 16,1$ of the 2 medical schools with mandatory palliative care clinical rotations was able to offer these rotations to only $71 \%$ of undergraduate students, for unknown reasons. Previous years (2007/08 to 2010/11) are not represented graphically because of insufficient data (see Appen- dix 2, Supplemental Table S1, available at www.cmajopen. ca/content/8/2/E257/suppl/DC1, for details).

\section{Postgraduate trainees}

\section{Enhanced Skills program}

In 2017/18, 13 medical schools (76\%) offered Enhanced Skills programs in palliative care leading to the College of Family Physicians of Canada Certificate of Added Competence in Palliative Care for family physicians. ${ }^{10}$ This is typically a 1-year program at the end of a 2 -year family medicine residency.

According to annual reports of the Canadian Post-M.D. Education Registry, between 2007/08 and 2017/18, 208 family medicine residents completed an Enhanced Skills program in palliative care (Table 1). Despite a lower number of trainees in 2011/12 and 2012/13, the number gradually increased over the study period, from 9 to 24 .

\section{Selected specialty programs}

Data for the postgraduate level were received from 13 medical schools for at least 1 year of the period 2007/08-2017/18. Of the 13 schools, 1 provided combined data for $2007 / 08$ 2016/17, and another provided data for 2016/17 only. Overall, data concerning palliative care clinical rotations were obtained from medical schools accounting for 41292 trainees in specialty or subspecialty programs in family medicine (13 schools), anesthesiology (12 schools), geriatric medicine

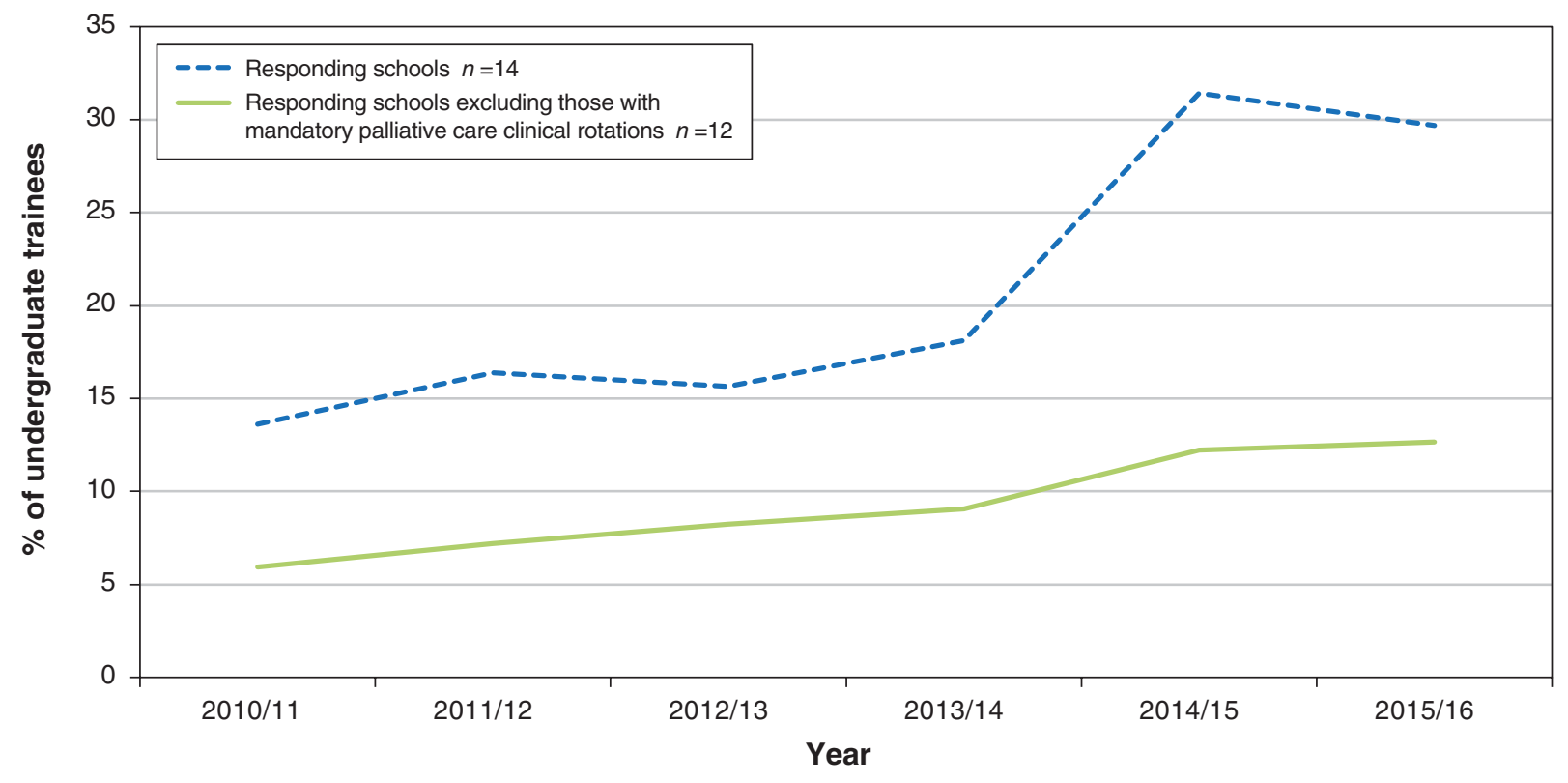

Figure 1: Estimated proportion of undergraduate trainees who completed palliative care clinical rotations at responding Canadian medical schools $(n=14)$ and at responding schools excluding those with mandatory palliative care clinical rotations $(n=12), 2010 / 11$ to $2015 / 16$. 
Table 1: Number of family medicine trainees who completed Enhanced Skills programs in palliative care, 2007/08 to 2017/18

\begin{tabular}{|c|c|c|c|c|c|c|c|c|c|c|c|c|}
\hline \multirow[b]{2}{*}{$\begin{array}{l}\text { Geographic } \\
\text { regiont }\end{array}$} & \multicolumn{12}{|c|}{ Year; no. of palliative care trainees* } \\
\hline & $\begin{array}{c}2007 / \\
08\end{array}$ & $\begin{array}{c}2008 / \\
09\end{array}$ & $\begin{array}{c}2009 / \\
10\end{array}$ & $\begin{array}{c}2010 / \\
11\end{array}$ & $\begin{array}{c}2011 / \\
12\end{array}$ & $\begin{array}{c}2012 / \\
13\end{array}$ & $\begin{array}{c}2013 / \\
14\end{array}$ & $\begin{array}{c}2014 / \\
15\end{array}$ & $\begin{array}{c}2015 / \\
16\end{array}$ & $\begin{array}{c}2016 / \\
17\end{array}$ & $\begin{array}{c}2017 / \\
18\end{array}$ & Total \\
\hline British Columbia & 2 & 3 & 3 & 2 & 2 & 2 & 2 & 1 & 4 & 3 & 0 & 24 \\
\hline Prairies & 4 & 4 & 6 & 6 & 3 & 2 & 5 & 4 & 5 & 4 & 5 & 48 \\
\hline Ontario & 3 & 3 & 5 & 10 & 8 & 3 & 12 & 12 & 12 & 13 & 14 & 95 \\
\hline Quebec & 0 & 3 & 2 & 5 & 1 & 2 & 4 & 1 & 4 & 3 & 4 & 29 \\
\hline Atlantic Canada & 0 & 1 & 1 & 1 & 2 & 2 & 1 & 0 & 2 & 1 & 1 & 12 \\
\hline Total & 9 & 14 & 17 & 24 & 16 & 11 & 24 & 18 & 27 & 24 & 24 & 208 \\
\hline
\end{tabular}

(11 schools), internal medicine (13 schools), neurology (9 schools) and psychiatry (11 schools), which corresponds to $68.9 \%$ of all residents in these specialties or subspecialties in all Canadian medical schools for the same period (see Appendix 2, Supplemental Table S2 for details).

Among the selected specialty or subspecialty training programs, 7505 palliative care clinical rotations were performed during training (Table 2). Psychiatry (64.5\%), geriatric medicine $(64.4 \%)$ and family medicine $(57.9 \%)$ had the highest estimated proportion of residents who completed a dedicated palliative care clinical rotation. In contrast, about 1 of 3 trainees in internal medicine (30.9\%) and neurology (28.2\%) completed a rotation in palliative care during their residency. Overall, the number of palliative care clinical rotations increased steadily over the study period for most of the specialty or subspecialty programs.

Table 3 presents the estimated proportion of family medicine residents who performed palliative care clinical rotations by medical school and by year. A total of 5488 palliative care clinical rotations were performed by family medicine trainees

Table 2: Number of palliative care clinical rotations and estimated proportion of residents completing such rotations by medical specialty or subspecialty and year, $2007 / 08$ to $2016 / 17$

\begin{tabular}{|c|c|c|c|c|c|c|c|c|c|c|c|}
\hline \multirow[b]{2}{*}{$\begin{array}{l}\text { Specialty/ } \\
\text { subspecialty }\end{array}$} & \multicolumn{11}{|c|}{ Year; no. of rotations in each specialty (estimated \% of residents who completed a dedicated rotation) } \\
\hline & $\begin{array}{c}2007 / \\
08\end{array}$ & $\begin{array}{c}2008 / \\
09\end{array}$ & $\begin{array}{c}2009 / \\
10\end{array}$ & $\begin{array}{c}2010 / \\
11\end{array}$ & $\begin{array}{c}2011 / \\
12\end{array}$ & $\begin{array}{c}2012 / \\
13\end{array}$ & $\begin{array}{c}2013 / \\
14\end{array}$ & $\begin{array}{c}2014 / \\
15\end{array}$ & $\begin{array}{c}2015 / \\
16\end{array}$ & $\begin{array}{c}2016 / \\
17\end{array}$ & Total $^{*}[\%] \dagger$ \\
\hline Family medicine & $\begin{array}{c}208 \\
(44.4)\end{array}$ & $\begin{array}{c}213 \\
(38.7)\end{array}$ & $\begin{array}{c}253 \\
(42.4)\end{array}$ & $\begin{array}{c}337 \\
(45.9)\end{array}$ & $\begin{array}{c}373 \\
(48.4)\end{array}$ & $\begin{array}{c}408 \\
(50.2)\end{array}$ & $\begin{array}{c}474 \\
(52.4)\end{array}$ & $\begin{array}{c}496 \\
(51.6)\end{array}$ & $\begin{array}{c}540 \\
(54.4)\end{array}$ & $\begin{array}{c}652 \\
(54.8)\end{array}$ & $\begin{array}{c}5488 \\
(57.9)[69.9]\end{array}$ \\
\hline \multicolumn{12}{|l|}{ Other } \\
\hline Anesthesiology & $\begin{array}{c}24 \\
(49.8)\end{array}$ & $\begin{array}{c}23 \\
(41.2)\end{array}$ & $\begin{array}{c}22 \\
(36.1)\end{array}$ & $\begin{array}{c}26 \\
(35.9)\end{array}$ & $\begin{array}{c}20 \\
(27.2)\end{array}$ & $\begin{array}{c}33 \\
(46.9)\end{array}$ & $\begin{array}{c}49 \\
(62.0)\end{array}$ & $\begin{array}{c}42 \\
(54.0)\end{array}$ & $\begin{array}{c}26 \\
(34.6)\end{array}$ & $\begin{array}{c}23 \\
(30.1)\end{array}$ & $\begin{array}{c}321 \\
(34.2)[68.2]\end{array}$ \\
\hline Geriatric medicine & $\begin{array}{c}2 \\
(30.8)\end{array}$ & $\begin{array}{c}0 \\
(0.0)\end{array}$ & $\begin{array}{c}3 \\
(60.0)\end{array}$ & $\begin{array}{c}3 \\
(50.0)\end{array}$ & $\begin{array}{c}3 \\
(75.0)\end{array}$ & $\begin{array}{c}6 \\
(92.3)\end{array}$ & $\begin{array}{c}11 \\
(88.0)\end{array}$ & $\begin{array}{c}9 \\
(60.0)\end{array}$ & $\begin{array}{c}11 \\
(66.7)\end{array}$ & $\begin{array}{c}15 \\
(68.2)\end{array}$ & $\begin{array}{c}87 \\
(64.4) \text { [91.3] }\end{array}$ \\
\hline Internal medicine & $\begin{array}{c}22 \\
(18.4)\end{array}$ & $\begin{array}{c}28 \\
(18.2)\end{array}$ & $\begin{array}{c}36 \\
(20.9)\end{array}$ & $\begin{array}{c}53 \\
(27.4)\end{array}$ & $\begin{array}{c}55 \\
(27.5)\end{array}$ & $\begin{array}{c}70 \\
(35.4)\end{array}$ & $\begin{array}{c}75 \\
(34.6)\end{array}$ & $\begin{array}{c}93 \\
(42.6)\end{array}$ & $\begin{array}{c}85 \\
(37.9)\end{array}$ & $\begin{array}{c}98 \\
(35.8)\end{array}$ & $\begin{array}{c}766 \\
(30.9)[68.9]\end{array}$ \\
\hline Neurology & $\begin{array}{c}8 \\
(55.6)\end{array}$ & $\begin{array}{c}6 \\
(33.3)\end{array}$ & $\begin{array}{c}6 \\
(29.1)\end{array}$ & $\begin{array}{c}12 \\
(49.6)\end{array}$ & $\begin{array}{c}7 \\
(26.1)\end{array}$ & $\begin{array}{c}9 \\
(32.8)\end{array}$ & $\begin{array}{c}9 \\
(33.1)\end{array}$ & $\begin{array}{c}8 \\
(32.0)\end{array}$ & $\begin{array}{c}15 \\
(54.3)\end{array}$ & $\begin{array}{c}7 \\
(26.5)\end{array}$ & $\begin{array}{c}92 \\
(28.2)[61.6]\end{array}$ \\
\hline Psychiatry & $\begin{array}{c}15 \\
(24.8)\end{array}$ & $\begin{array}{c}27 \\
(37.6)\end{array}$ & $\begin{array}{c}25 \\
(33.5)\end{array}$ & $\begin{array}{c}26 \\
(33.2)\end{array}$ & $\begin{array}{c}34 \\
(46.7)\end{array}$ & $\begin{array}{c}37 \\
(49.3)\end{array}$ & $\begin{array}{c}46 \\
(52.8)\end{array}$ & $\begin{array}{c}58 \\
(63.5)\end{array}$ & $\begin{array}{c}59 \\
(60.8)\end{array}$ & $\begin{array}{c}58 \\
(47.4)\end{array}$ & $\begin{array}{c}751 \\
(64.5)[67.8]\end{array}$ \\
\hline Subtotal & 71 & 84 & 92 & 120 & 119 & 155 & 190 & 210 & 196 & 201 & $\begin{array}{l}2017 \\
{[68.1]}\end{array}$ \\
\hline Total & 279 & 297 & 345 & 457 & 492 & 563 & 664 & 706 & 736 & 853 & $\begin{array}{l}7505 \\
{[68.9]}\end{array}$ \\
\hline
\end{tabular}


Table 3: Number of palliative care clinical rotations within family medicine and estimated proportion of residents performing such rotations by responding medical school and year, 2007/08 to 2016/17

\begin{tabular}{|c|c|c|c|c|c|c|c|c|c|c|c|}
\hline \multirow[b]{2}{*}{$\begin{array}{l}\text { Medical } \\
\text { school }\end{array}$} & \multicolumn{11}{|c|}{ Year*; no. of rotations in each school (estimated \% of family medicine residents who completed a dedicated rotation) } \\
\hline & $\begin{array}{c}2007 / \\
08\end{array}$ & $\begin{array}{c}2008 / \\
09\end{array}$ & $\begin{array}{c}2009 / \\
10\end{array}$ & $\begin{array}{c}2010 / \\
11\end{array}$ & $\begin{array}{c}2011 / \\
12\end{array}$ & $\begin{array}{c}2012 / \\
13\end{array}$ & $\begin{array}{c}2013 / \\
14\end{array}$ & $\begin{array}{c}2014 / \\
15\end{array}$ & $\begin{array}{c}2015 / \\
16\end{array}$ & $\begin{array}{c}2016 / \\
17 \dagger\end{array}$ & Total†‡ [\%]§ \\
\hline A & $\begin{array}{c}8 \\
(10.6)\end{array}$ & $\begin{array}{c}3 \\
(3.8)\end{array}$ & $\begin{array}{c}8 \\
(9.5)\end{array}$ & $\begin{array}{c}4 \\
(4.1)\end{array}$ & $\begin{array}{c}12 \\
(11.6)\end{array}$ & $\begin{array}{c}12 \\
(11.7)\end{array}$ & $\begin{array}{c}29 \\
(27.1)\end{array}$ & $\begin{array}{c}29 \\
(25.9)\end{array}$ & $\begin{array}{c}19 \\
(18.5)\end{array}$ & $\begin{array}{c}13 \\
(12.3)\end{array}$ & $\begin{array}{c}137 \\
(14.1)[100.0]\end{array}$ \\
\hline B & $\begin{array}{c}17 \\
(37.0)\end{array}$ & $\begin{array}{c}13 \\
(29.2)\end{array}$ & $\begin{array}{c}20 \\
(40.4)\end{array}$ & 18(33.0) & $\begin{array}{c}15 \\
(27.5)\end{array}$ & $\begin{array}{c}19 \\
(36.2)\end{array}$ & $\begin{array}{c}17 \\
(30.1)\end{array}$ & $\begin{array}{c}13 \\
(21.1)\end{array}$ & $\begin{array}{c}20 \\
(32.0)\end{array}$ & $\begin{array}{c}17 \\
(26.2)\end{array}$ & $\begin{array}{c}169 \\
(30.9)[100.0]\end{array}$ \\
\hline C & $\begin{array}{c}15 \\
(21.7)\end{array}$ & $\begin{array}{c}17 \\
(27.0)\end{array}$ & $\begin{array}{c}29 \\
(44.3)\end{array}$ & $\begin{array}{c}26 \\
(38.8)\end{array}$ & $\begin{array}{c}32 \\
(50.8)\end{array}$ & $\begin{array}{c}14 \\
(21.7)\end{array}$ & $\begin{array}{c}17 \\
(23.8)\end{array}$ & $\begin{array}{c}17 \\
(23.3)\end{array}$ & $\begin{array}{c}28 \\
(37.6)\end{array}$ & $\begin{array}{c}19 \\
(26.0)\end{array}$ & $\begin{array}{c}214 \\
(31.3)[100.0]\end{array}$ \\
\hline D & & & & $\begin{array}{c}29 \\
(35.6)\end{array}$ & $\begin{array}{c}29 \\
(31.7)\end{array}$ & $\begin{array}{c}25 \\
(27.9)\end{array}$ & $\begin{array}{c}38 \\
(46.9)\end{array}$ & $\begin{array}{c}32 \\
(34.4)\end{array}$ & $\begin{array}{c}54 \\
(51.7)\end{array}$ & $\begin{array}{c}38 \\
(34.7)\end{array}$ & $\begin{array}{c}245 \\
(37.7) \text { [75.0] }\end{array}$ \\
\hline $\mathrm{E}$ & & $\begin{array}{c}11 \\
(40.7)\end{array}$ & $\begin{array}{c}10 \\
(26.0)\end{array}$ & $\begin{array}{c}9 \\
(20.2)\end{array}$ & $\begin{array}{c}13 \\
(33.8)\end{array}$ & $\begin{array}{c}12 \\
(30.0)\end{array}$ & $\begin{array}{c}16 \\
(43.8)\end{array}$ & $\begin{array}{c}20 \\
(51.3)\end{array}$ & $\begin{array}{c}22 \\
(52.4)\end{array}$ & $\begin{array}{c}20 \\
(45.5)\end{array}$ & $\begin{array}{c}133 \\
(38.0)[96.8]\end{array}$ \\
\hline $\mathrm{F}$ & & & & & & & $\begin{array}{c}21 \\
(36.8)\end{array}$ & $\begin{array}{c}24 \\
(38.1)\end{array}$ & $\begin{array}{c}30 \\
(44.8)\end{array}$ & $\begin{array}{c}26 \\
(37.1)\end{array}$ & $\begin{array}{c}101 \\
(39.3)[49.8]\end{array}$ \\
\hline G & $\begin{array}{c}19 \\
(30.2)\end{array}$ & $\begin{array}{c}13 \\
(22.2)\end{array}$ & $\begin{array}{c}38 \\
(60.8)\end{array}$ & $\begin{array}{c}33 \\
(45.5)\end{array}$ & $\begin{array}{c}41 \\
(45.8)\end{array}$ & $\begin{array}{c}51 \\
(54.8)\end{array}$ & $\begin{array}{c}39 \\
(40.8)\end{array}$ & $\begin{array}{c}47 \\
(45.0)\end{array}$ & $\begin{array}{c}49 \\
(47.8)\end{array}$ & $\begin{array}{c}47 \\
(44.8)\end{array}$ & $\begin{array}{c}377 \\
(44.5)[100.0]\end{array}$ \\
\hline $\mathrm{H}$ & & $\begin{array}{c}0 \\
(0.0)\end{array}$ & $\begin{array}{c}0 \\
(0.0)\end{array}$ & $\begin{array}{c}26 \\
(34.7)\end{array}$ & $\begin{array}{c}27 \\
(33.3)\end{array}$ & $\begin{array}{c}33 \\
(37.5)\end{array}$ & $\begin{array}{c}55 \\
(64.3)\end{array}$ & $\begin{array}{c}63 \\
(72.0)\end{array}$ & $\begin{array}{c}71 \\
(77.6)\end{array}$ & $\begin{array}{c}70 \\
(68.6)\end{array}$ & $\begin{array}{c}345 \\
(47.5)[93.7]\end{array}$ \\
\hline I & $\begin{array}{c}48 \\
(48.7)\end{array}$ & $\begin{array}{c}44 \\
(43.3)\end{array}$ & $\begin{array}{c}45 \\
(42.9)\end{array}$ & $\begin{array}{c}74 \\
(66.7)\end{array}$ & $\begin{array}{c}75 \\
(68.2)\end{array}$ & $\begin{array}{c}90 \\
(72.3)\end{array}$ & $\begin{array}{c}88 \\
(63.8)\end{array}$ & $\begin{array}{c}93 \\
(62.2)\end{array}$ & $\begin{array}{c}97 \\
(59.9)\end{array}$ & $\begin{array}{c}108 \\
(68.1)\end{array}$ & $\begin{array}{c}762 \\
(60.5)[100.0]\end{array}$ \\
\hline J & $\begin{array}{c}43 \\
(85.1)\end{array}$ & $\begin{array}{c}50 \\
(93.5)\end{array}$ & $\begin{array}{c}49 \\
(86.7)\end{array}$ & $\begin{array}{c}58 \\
(93.5)\end{array}$ & $\begin{array}{c}69 \\
(104.5)\end{array}$ & $\begin{array}{c}82 \\
(101.9)\end{array}$ & $\begin{array}{c}76 \\
(81.7)\end{array}$ & $\begin{array}{c}82 \\
(88.2)\end{array}$ & $\begin{array}{c}79 \\
(81.0)\end{array}$ & $\begin{array}{c}74 \\
(76.3)\end{array}$ & $\begin{array}{c}662 \\
(88.3)[100.0]\end{array}$ \\
\hline K & $\begin{array}{c}58 \\
(86.6)\end{array}$ & $\begin{array}{c}62 \\
(86.1)\end{array}$ & $\begin{array}{c}54 \\
(77.1)\end{array}$ & $\begin{array}{c}60 \\
(87.0)\end{array}$ & $\begin{array}{c}60 \\
(82.2)\end{array}$ & $\begin{array}{c}70 \\
(89.2)\end{array}$ & $\begin{array}{c}78 \\
(93.4)\end{array}$ & $\begin{array}{c}76 \\
(89.4)\end{array}$ & $\begin{array}{c}71 \\
(81.6)\end{array}$ & $\begin{array}{c}89 \\
(109.9)\end{array}$ & $\begin{array}{c}678 \\
(88.5)[100.0]\end{array}$ \\
\hline Lף & & & & & & & & & & & $\begin{array}{c}1534 \\
(102.2)[100.0]\end{array}$ \\
\hline M & & & & & & & & & & $\begin{array}{c}131 \\
(73.4)\end{array}$ & $\begin{array}{c}13 \\
(73.4)[13.6]\end{array}$ \\
\hline Total [\%] & $\begin{array}{c}208 \\
(44.4) \\
{[55.4]}\end{array}$ & $\begin{array}{c}213 \\
(38.7) \\
{[62.6]}\end{array}$ & $\begin{array}{c}253 \\
(42.4) \\
{[63.0]}\end{array}$ & $\begin{array}{c}337 \\
(45.9) \\
{[71.6]}\end{array}$ & $\begin{array}{c}373 \\
(48.4 \\
{[70.2]}\end{array}$ & $\begin{array}{c}408 \\
(50.2) \\
{[70.2]}\end{array}$ & $\begin{array}{c}474 \\
(52.4) \\
{[73.9]}\end{array}$ & $\begin{array}{c}496 \\
(51.6) \\
{[74.7]}\end{array}$ & $\begin{array}{c}540 \\
(54.4) \\
{[74.5]}\end{array}$ & $\begin{array}{c}652 \\
(54.8) \\
{[87.5]}\end{array}$ & $\begin{array}{c}5488 \\
(57.9)[69.9]\end{array}$ \\
\hline $\begin{array}{l}\text { *Years for } \\
\dagger \text { Estimated } \\
\ddagger \text { Total for a } \\
\S[\%]=\text { perc } \\
\text { Supplemen } \\
\text { ๆMedical sc }\end{array}$ & $\begin{array}{l}\text { of family } \\
\text { e S2 for } \\
\text { provided }\end{array}$ & $\begin{array}{l}\text { dicine re } \\
\text { ails). } \\
\text { mbined }\end{array}$ & s for who & were avai & e/total nu & rof fam & dicine & ts, exp & as a pe & ge (see & ndix 2 \\
\hline
\end{tabular}

between 2007/08 and 2016/17, which corresponds to an estimated $57.9 \%$ of family medicine residents (range $14.1 \%-$ 102.2\%) (see Appendix 2, Supplemental Figure S1 for details).

\section{Interpretation}

Although most Canadian medical schools offered clinical rotations in palliative care, only a minority of trainees at the undergraduate and postgraduate levels completed such a rotation during their training.

The low rate of completion of palliative care rotations is not unique to Canada; it has also been observed in the United Kingdom, ${ }^{11,12}$ the United States, ${ }^{13}$ Switzerland ${ }^{14}$ and Thailand. ${ }^{15}$ However, this does not justify the status quo. In 2018 in Canada, cancer, heart and pulmonary diseases, cerebrovas- cular disease, diabetes mellitus, Alzheimer disease and chronic liver disease were among the leading causes of death. ${ }^{16}$ All are usually preceded by prolonged and chronic illnesses requiring symptom management and other psychosocial interventions to improve quality of life. Physicians who have no clinical experience with palliative care may lack the necessary skills and training to care for the majority of Canadians who will die from incurable chronic illnesses.

Adequate human and financial resources are lacking for palliative care training programs in most Canadian medical schools, which limits the opportunity for trainees to access palliative care training. ${ }^{17}$ Even if palliative care rotations were mandatory for the selected specialty or subspecialty programs in this study, current palliative care training resources would not be sufficient to meet this requirement at most centres. 
In a comparative study of palliative care education between the UK and the US, Hammel and colleagues ${ }^{18}$ suggested the creation of academic palliative care departments to develop and sustain this field through education and exposure to role models. Palliative care training has been recognized as an essential component of training for physicians for more than 20 years. ${ }^{19,20}$ Studies show that it positively influences students' ability to provide good clinical care ${ }^{21}$ and improves skills in end-of-life discussions. ${ }^{22}$ Palliative care training is also highly appreciated by medical students worldwide. ${ }^{11,23,24}$

The new 2-year subspecialty training programs in palliative medicine with corresponding recognition by the Royal College of Physicians and Surgeons of Canada may encourage the development of stronger palliative care programs in all Canadian medical schools. The College of Physicians of Quebec has yet to recognize this subspecialty. In the US, Congress recently passed the Palliative Care and Hospice Education and Training Act, ${ }^{25}$ which will increase the number of permanent faculty in palliative care. We hope this will provide impetus to improve palliative care education and research in Canada.

\section{Limitations}

Our study has limitations. We could not obtain data from all medical schools, and the relevant data were not kept by the College of Family Physicians of Canada or the Royal College of Physicians and Surgeons of Canada. The exclusion of these missing data from the analysis likely led to overestimation of the undergraduate and family medicine trainees who completed clinical rotations in palliative care, since the schools and programs with mandatory rotations would have had a greater influence on the overall number. Owing to the manner in which the data were compiled at each medical school, collecting data at the individual trainee level was not feasible, and we could only estimate the proportion of trainees who completed a palliative care rotation. If a trainee completed more than 1 rotation in palliative care, this trainee was counted multiple times in the numerator, which led to overestimation in some cases. Moreover, most medical schools did not record the exact duration of the rotations.

Notably, we also did not collect data on palliative care clinical training that may have occurred during other rotations, as some schools may have integrated some palliative care training within other rotations. During the study period, national palliative care competencies were defined for the undergraduate level but were not implemented in the curricula of all medical schools.

At the postgraduate level, at the time of the study, the majority of training programs did not have palliative care competencies. A description of standardized competencies in palliative care for all postgraduate specialty and subspecialty training programs in disciplines other than palliative medicine was subsequently developed in $2019 .{ }^{26} \mathrm{~A}$ set of 12 Entrustable Professional Activities for palliative medicine has also been developed, ${ }^{26}$ but these were meant to apply only to palliative care training programs, and they were not available during the study period.

\section{Conclusion}

Our study shows that, among Canadian medical trainees at the undergraduate and postgraduate levels, completion of palliative care clinical rotations is limited and inconsistent. Basic palliative competencies are essential for the provision of care in family medicine and in many specialties and subspecialties. It is well recognized that, without dedicated clinical exposure to palliative care, many trainees will enter practice without these vital competencies required for optimal care of the growing population of patients with chronic incurable illnesses and at the end of life.

\section{References}

1. Lorenz KA, Lynn J, Dy SM, et al. Evidence for improving palliative care at the end of life: a systematic review. Ann Intern Med 2008;148:147-59.

2. Temel JS, Greer JA, Muzikansky A, et al. Early palliative care for patients with metastatic non-small-cell lung cancer. N Engl f Med 2010;363:733-42.

3. Zimmermann C, Swami N, Krzyzanowska M, et al. Early palliative care for patients with advanced cancer: a cluster-randomised controlled trial. Lancet 2014;383:1721-30.

4. Bakitas M, Lyons KD, Hegel MT, et al. Effects of a palliative care intervention on clinical outcomes in patients with advanced cancer: the Project ENABLE II randomized controlled trial. 7 AMA 2009;302:741-9.

5. Palliative care: a vital service with clear economic, health and social benefits. Surrey (BC): Canadian Society of Palliative Care Physicians; 2017. Available: www. cspcp.ca/wp-content/uploads/2017/02/Economics-of-Palliative-Care-Final-EN. pdf (accessed 2018 Sept. 14).

6. The way forward - moving towards an integrated palliative approach to care: survey of GP/FPs and nurses in primary care. Ottawa: Canadian Hospice Palliative Care Association; 2014.

7. Policy Resolution GC13-71 - Training in palliative care. Ottawa: Canadian Medical Association; 2013. Available: https://policybase.cma.ca/documents/ Policypdf/PD16-01.pdf (accessed 2018 Sept. 14).

8. How to improve palliative care in Canada: a call to action for federal, provincial, territorial, regional and local decision-makers. Surrey (BC): Canadian Society of Palliative Care Physicians; 2016. Available: www.cspcp.ca/wp-content/uploads/2016/11/ Full-Report-How-to-Improve-Palliative-Care-in-Canada-FINAL-Nov-2016.pdf (accessed 2018 Sept. 14).

9. Data. Association of Faculties of Medicine of Canada. Available: https://afmc. $\mathrm{ca} / \mathrm{en} /$ faculties/data (accessed 2018 May 1).

10. Individual specialty reports. Ottawa: Canadian Post-M.D. Education Registry. Available: https://caper.ca/postgraduate-medical-education/individual -specialty-reports (accessed 2018 May 1).

11. Walker S, Gibbins J, Paes P, et al. Palliative care education for medical students: differences in course evolution, organisation, evaluation and funding: a survey of all UK medical schools. Palliat Med 2017;31:575-81.

12. Walker S, Gibbins J, Barclay S, et al. Progress and divergence in palliative care education for medical students: a comparative survey of UK course structure, content, delivery, contact with patients and assessment of learning. Palliat Med 2016;30:834-42.

13. Horowitz R, Gramling R, Quill T. Palliative care education in U.S. medical schools. Med Educ 2014;48:59-66.

14. Pereira J, Pautex S, Cantin B, et al. Palliative care education in Swiss undergraduate medical curricula: a case of too little, too early. Palliat Med 2008;22:730-5.

15. Suvarnabhumi K, Sowanna N, Jiraniramai S, et al. Situational analysis of palliative care education in Thai medical schools. Palliat Care 2013;7:25-9.

16. Table 13-10-0394-01 Leading causes of death, total population, by age group. Ottawa: Statistics Canada; updated 2020 Jan. 6. Available: https://www150. statcan.gc.ca/t1/tbl1/en/tv.action?pid=1310039401 (accessed 2020 Jan. 16).

17. Boyle A, Downar J, Herx L, et al. CSPCP Special Session (Part I): Current educational approaches to palliative care in Canada [oral presentation]. 13th Annual Advanced Learning in Palliative Medicine Conference; 2017 June 1-3; Edmonton $(\mathrm{AB})$.

18. Hammel JF, Sullivan AM, Block SD, et al. End-of-life and palliative care education for final-year medical students: a comparison of Britain and the United States. 7 Palliat Med 2007;10:1356-66.

19. Oneschuk D. Undergraduate medical palliative care education: a new Canadian perspective. 7 Palliat Med 2002;5:43-7.

20. MacDonald N, Boisvert M, Dudgeon D, et al. The Canadian Palliative Care Education Group. 7 Palliat Care 2000;16:13-5.

21. Centeno C, Rodriguez-Nunez A. The contribution of undergraduate palliative care education: Does it influence the clinical patient's care? Curr Opin Support Palliat Care 2015;9:375-91.

22. McKinlay E, McBain L, Stanley J, et al. Does a multi component palliative care education programme help medical students talk with patients at end-of-life? Med Teach 2011;33:864. 
23. Centeno C, Ballesteros M, Carrasco JM, et al. Does palliative care education matter to medical students? The experience of attending an undergraduate course in palliative care. BM7 Support Palliat Care 2016;6:128-34.

24. Mutto EM, Bunge S, Vignaroli E, et al. Medical students' palliative care education in a Latin American university: a three-year experience at Austral University in Buenos Aires, Argentina. 7 Palliat Med 2014;17:1137-42.

25. Palliative Care and Hospice Education and Training Act H.R.647 - 116th Congress (2019-2020). Available: https://www.congress.gov/bill/116th-congress/ house-bill/647/text (accessed 2020 Jan. 16).

26. PACE competencies - postgrad: postgrad competencies for palliative approach to care. Surrey (BC): Canadian Society of Palliative Care Physicians; 2019. Available: https://www.cspcp.ca/information/pace-competencies-postgrad/ (accessed 2020 Mar. 30).

27. Myers J, Krueger P, Webster F, et al. Development and validation of a set of palliative medicine entrustable professional activities: findings from a mixed methods study. 7 Palliat Med 2015;18:682-90.

Affiliations: Faculty of Medicine (Gagnon), Laval University; Research Centre of the Centre hospitalier universitaire de Québec-Université Laval (Gagnon, Jolicoeur, Labonté), Québec, Que.; Canadian Society of Palliative Care Physicians (Boyle, Taylor, Downar), Surrey, BC; Department of Family Medicine (Boyle), McMaster University, Hamilton, Ont.; Department of Medicine (Downar), University of Ottawa, Ottawa, Ont.

Contributors: Bruno Gagnon, Anne Boyle, Fabienne Jolicoeur, Kim Taylor and James Downar contributed to the study conception and design. Bruno Gagnon and Fabienne Jolicoeur collected and analyzed the data. All of the authors were involved in data interpretation, drafted the manuscript and revised it critically for important intellectual content, approved the final version to be published and agreed to be accountable for all aspects of the work.

Funding: This work was supported by the Canadian Society of Palliative Care Physicians and by unrestricted educational grants from Innovative Medicines Canada and Purdue Pharma.

Data sharing: The data were obtained from medical schools, and their permission to share these data was not obtained.

Supplemental information: For reviewer comments and the original submission of this manuscript, please see www.cmajopen.ca/content/8/2/ E257/suppl/DC1. 